\title{
Rovibronic spectra of molecules dressed by light fields
}

\author{
Tamás Szidarovszky ๑* and Attila G. Császár \\ Laboratory of Molecular Structure and Dynamics, Institute of Chemistry, ELTE Eötvös Loránd University \\ and MTA-ELTE Complex Chemical Systems Research Group, Pázmány Péter sétány 1/A, H-1117 Budapest, Hungary
}

Gábor J. Halász

Department of Information Technology, University of Debrecen, P.O. Box 400, H-4002 Debrecen, Hungary

Ágnes Vibók ${ }^{\dagger}$

Department of Theoretical Physics, University of Debrecen, P.O. Box 400, H-4002 Debrecen, Hungary and ELI-ALPS, ELI-HU Non-Profit, Ltd., Dugonics tér 13, H-6720 Szeged, Hungary

(Received 3 April 2019; revised manuscript received 19 June 2019; published 19 September 2019)

\begin{abstract}
The theory of rovibronic spectroscopy of light-dressed molecules is presented within the framework of quantum mechanically treated molecules interacting with classical light fields. The numerical application involves the homonuclear diatomic molecule $\mathrm{Na}_{2}$, for which the general formulas can be simplified considerably and the physical processes leading to the light-dressed spectra can be understood straightforwardly. The physical origin of different peaks in the light-dressed spectrum of $\mathrm{Na}_{2}$ is given and the light-dressed spectrum is investigated in terms of its dependence on the dressing field's intensity and wavelength, the turn-on time of the dressing field, and the temperature. The important implications of light-dressed spectroscopy on deriving field-free spectroscopic quantities are also discussed.
\end{abstract}

DOI: 10.1103/PhysRevA.100.033414

\section{INTRODUCTION}

Atomic and molecular spectroscopy are among the most successful tools of science both in fundamental research and in practical applications. Despite the 200-year-long history of spectroscopy, new approaches and methods are being developed to this day [1]. This is strongly related to the remarkable advances in the available experimental techniques and light sources. Some notable developments include frequency-comb techniques [2-5], facilitating extremely precise and accurate measurements in the frequency domain, and the ability to generate ultrashort and intense laser pulses [6,7], allowing for time-resolved spectroscopies on femtosecond or even attosecond $[7,8]$ timescales.

A common approach in the application of spectroscopic techniques is the use of two (or more) light pulses, with some pulses acting as so-called pump pulses, which induce specific changes in the system, while subsequent so-called probe pulses are used (directly or indirectly) to measure the changes induced by the pump pulse(s). If the durations of both the pump and the probe pulses are short, then repeating the experiment with varying time delays between the pulses can lead to time-resolved dynamical information $[9,10]$ or by means of a Fourier transformation to multidimensional and/or high-resolution spectra [11,12]. If the pump and probe pulses overlap in time, the signal recorded by the probe pulse represents the so-called field-dressed or light-dressed

\footnotetext{
*tamas821@ caesar.elte.hu

†vibok@phys.unideb.hu
}

properties of the system [13-15], where the pump pulse acts as the dressing field. If both the pump and probe pulses are long with respect to the timescales of the processes investigated, then one obtains static spectral properties of the light-dressed system. We investigate the last scenario in this paper and henceforth call it light-dressed spectroscopy. The theoretical and experimental methods for investigating spectral transitions between atomic light-dressed states are well developed [13]. As to molecules, the concept of light-dressed electronic states, also called light-dressed potentials, has been utilized with success to understand nuclear dynamics both in experimental and in theoretical studies [16-22]. To some extent light-dressed rovibrational spectroscopy has been adopted for molecular systems, as well; for example, Autler-Townes-type splittings [23] of rotational transitions have been induced with microwave radiation to deduce molecular parameters $[24,25]$ or to promote the assignment of rovibrational spectra [26]. Related to light-dressed spectroscopy, the optical absorption of electronic materials driven away from equilibrium by laser light has also been investigated [27].

In a previous theoretical study of the present authors, in which all molecular degrees of freedom were incorporated into the concept of light-dressed states, the rovibronic spectrum of light-dressed $\mathrm{Na}_{2}$ was investigated in the context of how the presence of a light-induced conical intersection $[28,29]$, generated by the dressing field, can be identified in the spectrum [30,31]. The modeling work was carried out for both the classical dressing field of laser radiation [30] and the quantized dressing field of a microscopic cavity mode [31].

It is our firm belief that the spectroscopy of light-dressed molecules is of fundamental interest and could provide 
valuable scientific information on both light-dressed and fieldfree molecular systems. For example, because of the response of light-dressed systems to external fields is different from that of the corresponding field-free systems, understanding and predicting the absorption and emission properties of lightdressed molecules can be useful for tuning or controlling optical processes, such as those in coherent control schemes or laser cooling [32-36]. In this paper we provide an introduction to the theory of computing light-dressed spectra induced by classical light fields and investigate certain aspects and spectroscopic implications of light-dressed spectroscopy, such as the effects of dressing-light intensity and frequency and those of finite nonzero temperature and the turn-on time of the dressing field. A procedure to derive fieldfree transition frequencies from light-dressed spectra is also proposed.

\section{THE THEORETICAL APPROACH}

To make reading of this paper easier, the presentation of Floquet theory $[14,37,38]$ and some of the detailed derivations have been moved to the Appendices. The Appendices contain a large number of equations and only a few equations are given in this section. Those not familiar with Floquet theory or those interested in modeling details may want to read the Appendices before reading this section.

We advocate the use of a three-step theoretical approach to compute the light-dressed spectra of molecules. First, compute the field-free rovibronic states of the investigated molecule; second, using the field-free eigenstates as basis functions, compute the light-dressed states; and, finally, compute the transitions between the light-dressed states.

As to the physical scenario to be simulated, we make a couple of important assumptions.

(1) Initially the molecule is in a field-free eigenstate in the gas phase.

(2) The molecule is exposed to a medium-intensity dressing light $\left(I=10^{7}-5 \times 10^{9} \mathrm{~W} \mathrm{~cm}^{-2}\right.$ in the numerical examples of this paper), which is turned on adiabatically, i.e., its envelope varies much slower than the rovibronic timescales characterizing the molecule (such a dressing pulse converts the molecular wave function into a light-dressed state or a superposition of light-dressed states; see Appendices A and B). In the numerical examples considered below, the slowest molecular timescale is around 100 ps; thus, dressing pulses of at least nanosecond length could be considered as turning on adiabatically.

(3) The probe pulse, introduced to record the static rovibronic spectrum of the light-dressed molecule, is weak.

\section{A. Light-dressed states}

We determine the light-dressed states generated by the dressing light within the framework of Floquet theory
[14,37,38]. As detailed in Appendix A, in the presence of a dressing field periodic in time, the solution of the timedependent Schrödinger equation (TDSE) can be written as a superposition of light-dressed states (Floquet states) $\left|\Phi_{k}(t)\right\rangle$. With the initial wave function being a field-free eigenstate and the dressing field being turned on adiabatically, the generated light-dressed wave function is composed of a single lightdressed state (see Appendix B) $[39,40]$. Because the $\left|\Phi_{k}(t)\right\rangle$ states are periodic in time, they can be expanded as a Fourier series:

$$
\left|\Phi_{k}\right\rangle=\sum_{n, \alpha, v, J} C_{n, \alpha v J}^{(k)}|\alpha v J\rangle|n\rangle,
$$

where we have used the notation called Floquet-state nomenclature [37], in which $\langle t \mid n\rangle=e^{i n \omega_{1} t}$ and $\omega_{1}=2 \pi / T$ with $\left|\Phi_{k}(t+T)\right\rangle=\left|\Phi_{k}(t)\right\rangle$. In Eq. (1), $\alpha, v$, and $J$ represent electronic, vibrational, and rotational quantum numbers, respectively. The expansion coefficients $C_{n, \alpha v J}^{(k)}$ are obtained by diagonalizing the Floquet Hamiltonian of Eq. (A13).

In practical applications, when the Floquet Hamiltonian can be simplified to the form of Eq. (A18), i.e., when molecules have no permanent dipole and no intrinsic nonadiabatic couplings (or when the transitions originating from the permanent dipole and intrinsic nonadiabatic couplings can be neglected), and the dressing-field intensity is moderate enough to allow for the $2 \times 2$ Floquet Hamiltonian approach [41], light-dressed states have the simplified form of

$$
\left|\Phi_{k}(n)\right\rangle=\sum_{v, J} C_{X v J}^{(k)}|X v J\rangle|n\rangle+\sum_{v, J} C_{A v J}^{(k)}|A v J\rangle|n-1\rangle,
$$

where $X$ and $A$ represent the two electronic states considered (as usual, $X$ denotes the ground electronic state).

\section{B. Transitions between light-dressed states}

Once the light-dressed states have been determined, one can compute the transition probabilities between the different light-dressed states as induced by the weak probe pulse. Following the standard approach of molecular spectroscopy [42], we use first-order time-dependent perturbation theory (TDPT1), as detailed in Appendix C.

For physical scenarios in which the light-dressed states have the form shown in Eq. (2), such as the light-dressed states of the $\mathrm{Na}_{2}$ molecule investigated below, the $T_{l \leftarrow k}$ TDPT1 transition amplitude induced between the $k$ th and $l$ th light-dressed states by an interaction of the form $\hat{W}_{2}(t)=-\mathbf{E}_{2} \hat{\boldsymbol{\mu}} \cos \left(\omega_{2} t\right)$ gives [see Eq. (C18)]

$$
\begin{aligned}
T_{l \leftarrow k} \propto & \sum_{v, J} \sum_{v^{\prime}, J^{\prime}} C_{A v^{\prime} J^{\prime}}^{(l) *} C_{X v J}^{(k)}\left\langle A v^{\prime} J^{\prime}\left|\mathbf{E}_{2} \hat{\boldsymbol{\mu}}\right| X v J\right\rangle \delta\left(\varepsilon_{k}-\varepsilon_{l}-\hbar \omega_{1} \pm \hbar \omega_{2}\right) \\
& +\sum_{v, J} \sum_{v^{\prime}, J^{\prime}} C_{X v^{\prime} J^{\prime}}^{(l) *} C_{A v J}^{(k)}\left\langle X v^{\prime} J^{\prime}\left|\mathbf{E}_{2} \hat{\boldsymbol{\mu}}\right| A v J\right\rangle \delta\left(\varepsilon_{k}-\varepsilon_{l}+\hbar \omega_{1} \pm \hbar \omega_{2}\right),
\end{aligned}
$$


where $\mathbf{E}_{2}$ is the electric field vector of the probe pulse; $\hat{\boldsymbol{\mu}}$ is the molecular dipole operator; $\hbar \omega_{1}$ and $\hbar \omega_{2}$ are the photon energies of the dressing and probe fields, respectively; and $\varepsilon_{i}$ is the quasienergy of the $i$ th light-dressed state.

Based on the arguments of the delta functions in Eq. (3), the first term can be interpreted as a transition between lightdressed states having quasienergies $\varepsilon_{k}$ and $\varepsilon_{l}+\hbar \omega_{1}$, that is, a transition between $\left|\Phi_{k}(n)\right\rangle$ and $\left|\Phi_{l}\left(n^{\prime}\right)\right\rangle$ with $n=n^{\prime}-1$. Similarly, the second term in Eq. (3) can be interpreted as a transition between $\left|\Phi_{k}(n)\right\rangle$ and $\left|\Phi_{l}\left(n^{\prime}\right)\right\rangle$ with $n=n^{\prime}+1$.

\section{Computational details}

We choose to demonstrate the numerical application of the theory introduced above and in the Appendices on the $\mathrm{Na}_{2}$ molecule. In our simulations we consider the $\mathrm{X}^{1} \Sigma_{\mathrm{g}}^{+}$ ground and the first excited $\mathrm{A}^{1} \Sigma_{\mathrm{u}}^{+}$electronic states of $\mathrm{Na}_{2}$, for which we use the potential-energy curves (PEC) and the transition dipole function of Refs. [43] and [44], respectively. The field-free rovibrational eigenstates of $\mathrm{Na}_{2}$ on the $V_{X}(R)$ and $V_{A}(R)$ PECs are computed using 200 spherical-DVR basis function [45] with the related grid points placed in the internuclear coordinate range $(0,10)$ bohr, where DVR stands for discrete variable representation. All rovibrational eigenstates with $J<16$ and an energy not exceeding the zero-point energy of the respective PEC by more than $2000 \mathrm{~cm}^{-1}$ were included into the basis representing the Floquet Hamiltonian of Eq. (A18), which was then diagonalized to obtain the light-dressed states.

In all computations the polarization vector of the probe pulse was assumed to be parallel to the polarization vector of the pump pulse, meaning that the projection of the total angular momentum onto this axis is a conserved quantity during our simulations.

When investigating the effect of the turn-on time of the dressing field (see Sec. IIIE), the TDSE was solved using the simple formula $\boldsymbol{\Psi}(t+d t)=e^{-(i / \hbar) \mathbf{H}(t) d t} \boldsymbol{\Psi}(t)$. Due to the small size of $\mathbf{H}(t)$ (a few thousand by a few thousand) the exponential function could be constructed by diagonalizing $\mathbf{H}(t)$ at each time step.

\section{RESULTS AND DISCUSSION}

\section{A. Interpretation of the light-dressed spectrum}

Before investigating the light-dressed spectra in detail, it is worth considering their expected structure qualitatively. Naturally, the light-dressed spectrum strongly depends on the specific molecule investigated and the properties of the dressing field. For $\mathrm{Na}_{2}$, the rotational, vibrational, and electronic transition frequencies considered in this paper are around the order of 1, 100, and $15000 \mathrm{~cm}^{-1}$, respectively. Figure 1 demonstrates the landscape of light-dressed PECs for the $\mathrm{Na}_{2}$ molecule dressed by a $\lambda=657 \mathrm{~nm}$ wavelength light, which is near resonant with the transition between the $\mid \begin{array}{lll}X & 0 & 0\rangle\end{array}$ and $\left|\begin{array}{lll}A & 2 & 1\end{array}\right\rangle$ states. As can be seen in Fig. 1, the manifolds of light-dressed states, labeled by $n$, are well separated. Based on Eq. (3), arrows are drawn to indicate the physical origin of possible absorption and stimulated emission processes induced by the probe pulse. As seen in Fig. 1, absorption originates from the first term in Eq. (3), in which the initial

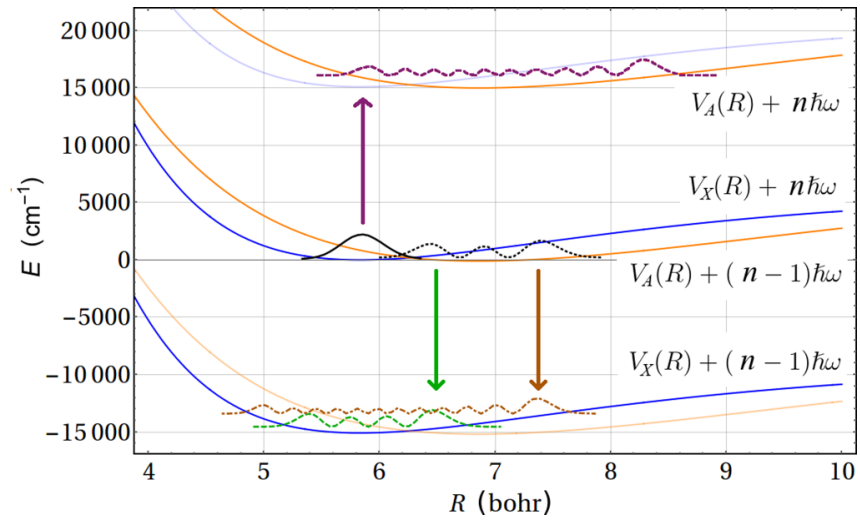

FIG. 1. Light-dressed diabatic PECs of $\mathrm{Na}_{2}$ obtained with a dressing-light wavelength of $\lambda=657 \mathrm{~nm}$, which is near resonant

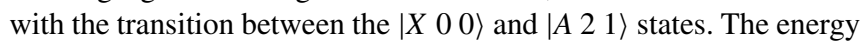
scale stands for quasienergy. Vibrational probability densities are

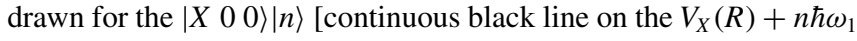
PEC], $|X \quad 30\rangle|n-1\rangle$ [green dashed line on the $V_{X}(R)+(n-1) \hbar \omega_{1}$ PEC], $|X \quad 110\rangle|n-1\rangle$ [brown dashed line on the $V_{X}(R)+(n-1)$ $\hbar \omega_{1}$ PEC], $|A 21\rangle|n-1\rangle$ [black dotted line on the $V_{A}(R)+(n-1)$ $\hbar \omega_{1}$ PEC], and $|A 91\rangle|n\rangle$ [purple dashed line on the $V_{A}(R)+n \hbar \omega_{1}$ PEC] states. Upward and downward pointing vertical arrows represent transitions of absorption and stimulated emission, respectively. The two product states with the largest contribution to the lightdressed state correlating to $|X \quad 00\rangle$ at $\left|E_{1}\right| \rightarrow 0$ are $|X \quad 0 \quad 0\rangle|n\rangle$ and $|A 21\rangle|n-1\rangle$.

light-dressed state contributes with its $X$ ground electronic state component and the final state contributes with its $A$ excited electronic state component. On the other hand, stimulated emission originates from the second term in Eq. (3), in which the initial light-dressed state contributes with its $A$ excited electronic state component and the final state contributes with its $X$ ground electronic state component.

\section{B. Intensity dependence of the light-dressed spectrum}

Figure 2 shows the absorption and stimulated emission spectra of $\mathrm{Na}_{2}$ at $0 \mathrm{~K}$, when the molecule is dressed by $657 \mathrm{~nm}$ wavelength light fields of different intensity. Note that there is no special reason for choosing $657 \mathrm{~nm}$ to demonstrate the intensity dependence of the light-dressed spectrum; it is merely a convenient choice for which the light-dressed PECs and some relevant states are already depicted in Fig. 1.

As seen in Fig. 2, with increasing dressing-field intensity the envelopes of both the absorption and the stimulated emission spectra increase. At the limit of zero dressing-field intensity, the stimulated emission peaks disappear, as expected. Note that the light-dressed states and the corresponding lightdressed spectra change if the dressing-field wavelength is changed. Therefore, if dressing-light wavelengths different from $657 \mathrm{~nm}$ are used, the envelope of the absorption spectrum might decrease or even show no monotonic behavior with increasing dressing-light intensity.

Inspecting the individual transition lines reveals that introducing the dressing field leads to the splitting of existing fieldfree absorption peaks as well as the appearance of new peaks, as shown in the upper and lower panels of Fig. 3, respectively. It is possible to understand and assign the transition peaks 

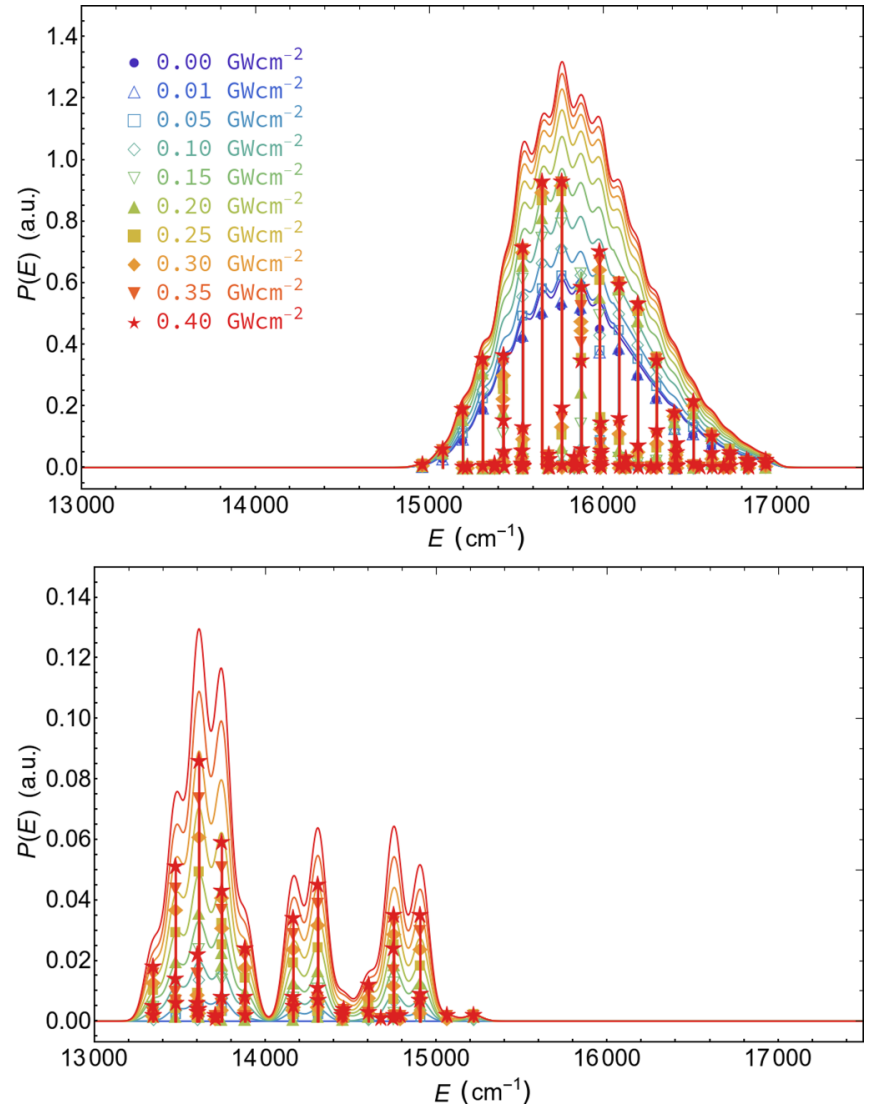

FIG. 2. Absorption (upper panel) and stimulated emission (lower panel) spectra of $\mathrm{Na}_{2}$ dressed with a $657 \mathrm{~nm}$ wavelength laser light at $0 \mathrm{~K}$. The stick spectra were computed using Eq. (3) and show transitions from the field-dressed state, which correlates to the $\mid \begin{array}{lll}X & 0 & 0\end{array}$ rovibronic ground state in the limit of the dressing-field intensity going to zero. The envelopes shown are obtained by taking the convolution of the stick spectra with a Gaussian function having a standard deviation of $\sigma=50 \mathrm{~cm}^{-1}$.

of the light-dressed spectrum based on the selection rules of transitions between field-free states and the fact that the light-dressed states can be described as a superposition of field-free states.

For example, the upper panel of Fig. 3 shows the progression of three peaks, corresponding to transitions from the initial state (light-dressed state correlating to the field-free ground state) composed primarily of the $\mid \begin{array}{lll}X & 0 & 0\rangle\end{array}$ state with smaller contributions from the $\left|\begin{array}{lll}X & 0 & 2\rangle\end{array}\right\rangle$ and $\left|\begin{array}{lll}A & 2 & 1\end{array}\right\rangle$ states to light-dressed states composed primarily of the $|A 71\rangle$, $|A 73\rangle$, and $|A 75\rangle$ states, with $|X v J\rangle$-type states ( $J$ even) contributing as well. These transitions can be interpreted as originating from the field-free transition $|A \quad 7 \quad 1\rangle \leftarrow\left|\begin{array}{lll}X & 0 & 0\end{array}\right\rangle$, which is split due to the mixing of field-free states through the light-matter coupling with the dressing field. Such peak splittings are similar in spirit to the well-known AutlerTownes effect [23] utilized in spectroscopy (see, for example, Refs. [24-26]). Furthermore, the upper panel of Fig. 3 not only demonstrates splitting of the peak, during which the sum of individual peak intensities remains unchanged, but exhibits an overall increase in the peak intensities when the strength of the dressing field is increased. In a spectroscopic context the
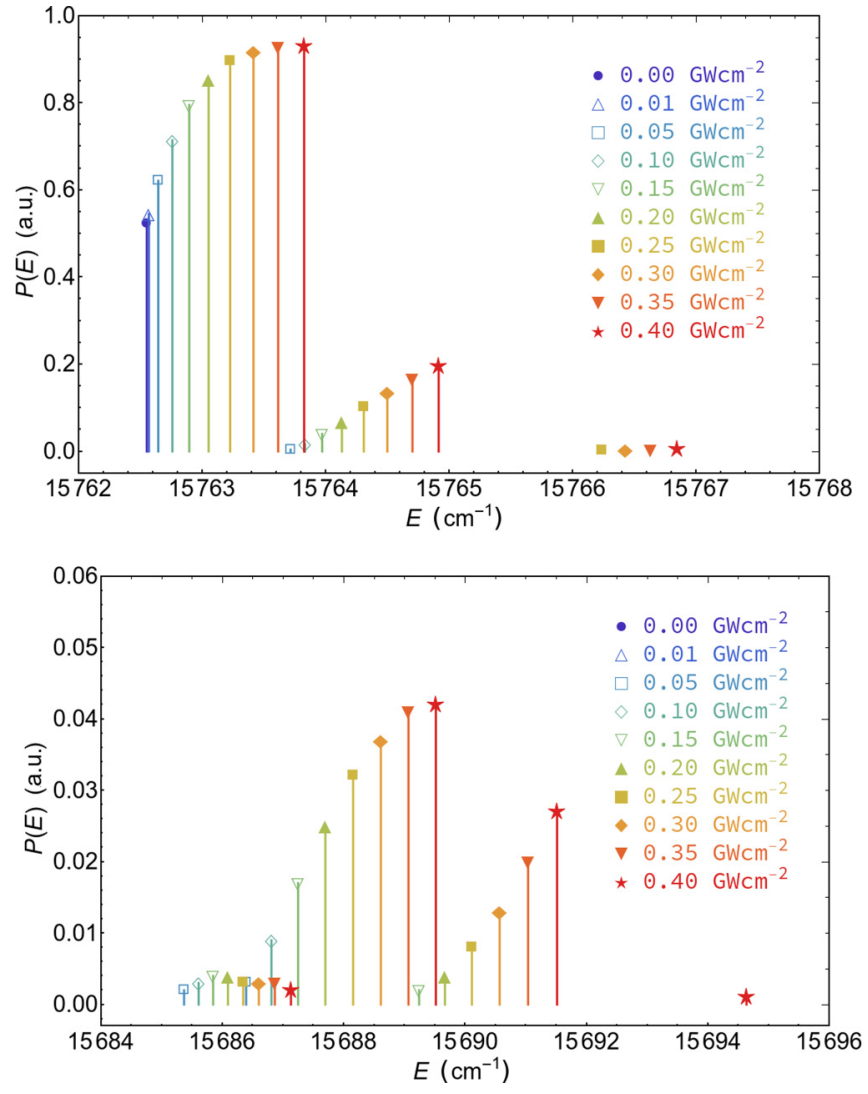

FIG. 3. Progression of selected light-dressed absorption peaks of $\mathrm{Na}_{2}$ dressed with a $657 \mathrm{~nm}$ wavelength laser light at $0 \mathrm{~K}$. The upper panel shows peaks originating from the field-free transition $|X \quad 00\rangle \rightarrow|A 71\rangle$, while the lower panel shows peaks which have no field-free counterpart.

change in transition peak intensities resulting from couplings between eigenstates of a zeroth-order Hamiltonian is usually called intensity borrowing [42].

The lower panel of Fig. 3 shows the progression of three peaks, which do not arise from the splitting of an existing field-free peak but appear as new peaks. These transitions occur between the initial state (light-dressed state correlating to the field-free ground state) composed primarily of $\mid \begin{array}{lll}X & 0 & 0\end{array}$ with smaller contributions from $\left|\begin{array}{llll}X & 0 & 2\rangle\end{array}\right\rangle$ and $\left.\mid \begin{array}{lll}A & 2 & 1\rangle\end{array}\right)$ and light-dressed states composed primarily of the $|X 30\rangle,|X 32\rangle$, and $|X 34\rangle$ states. Such transitions are forbidden in the limit of zero dressing-light intensity; however, they become visible as the light-matter coupling with the dressing field contaminates the $\mid \begin{array}{llll}X & 3 & J\rangle\end{array}$ states ( $J$ even) with $|A \quad v \quad 1\rangle$-type states, to which $\left|\begin{array}{llll}X & 0 & 0\end{array}\right\rangle$ has allowed transitions. The appearance of transition peaks as a result of such a mixing phenomenon can be understood as an intensity borrowing effect.

As to the stimulated emission peaks shown in the lower panel of Fig. 2, they represent transitions from the initial state to light-dressed states composed primarily of vibrationally highly excited $|X v 0\rangle$ - and $|X v 2\rangle$-type states, with $\left|A v^{\prime} J\right\rangle$ type states $(J$ odd $)$ contributing as well.

\section{Predicting field-free properties via extrapolation}

Although light-dressed spectroscopy might provide transition peaks forbidden in the field-free case, the transition 


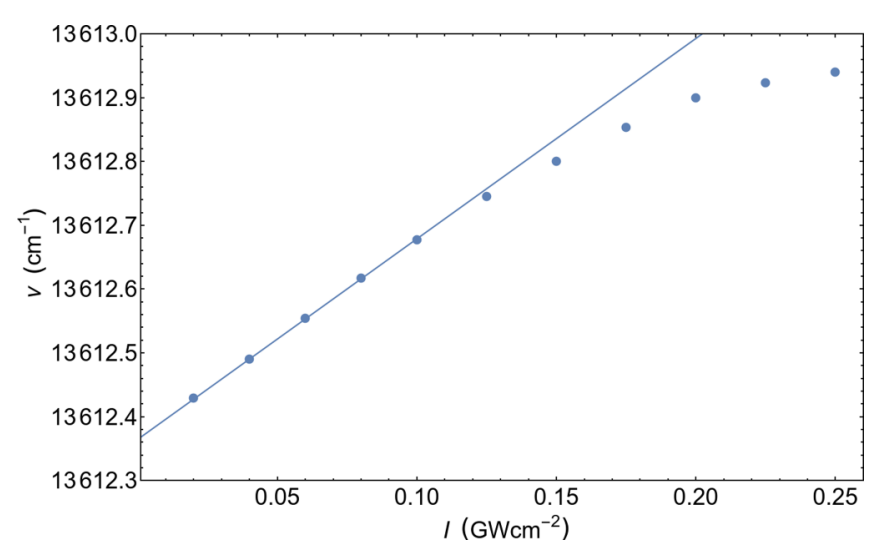

FIG. 4. Position of the stimulated emission peak near 13612.5 $\mathrm{cm}^{-1}$ as a function of dressing-light intensity for $\lambda=657 \mathrm{~nm}$. The straight line plotted was obtained by fitting a linear function to the first four data points.

frequencies between light-dressed states are in general different from the transition frequencies between field-free states. If one is interested in obtaining field-free transition frequencies, one might record the light-dressed spectrum at several dressing-field intensities and extrapolate to the zero intensity limit. Such a procedure is of course most valuable if the transition is forbidden in the field-free case.

As an example, we examine the stimulated emission peak at around $13612.5 \mathrm{~cm}^{-1}$ (see Fig. 4). The emission peak around $13612.5 \mathrm{~cm}^{-1}$ represents a transition in which the initial state is composed primarily of the $|X 00\rangle$ ground state with the $\left|\begin{array}{llll}X & 0 & 2\rangle\end{array}\right\rangle$ and $\mid \begin{array}{llll}A & 2 & 1\rangle\end{array}$ states contributing as well, and the final state is composed primarily of $|X 110\rangle$, with

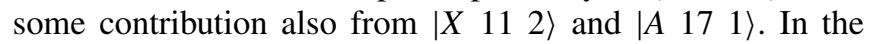
limit of zero dressing-light intensity, the initial and final states correlate to $\mid \begin{array}{lll}X & 0 & 0\rangle\end{array}$ and $|X \quad 110\rangle$, respectively. Transition between these two field-free states is forbidden; nonetheless, their accurate transition frequency can be obtained by extrapolating the light-dressed transition frequency to the limit of zero dressing-light intensity. As expected and seen in Fig. 4, linear extrapolation might be pursued if data points at low dressing-light intensities are used. On the other hand, by increasing the dressing-light intensity above a certain point, the relation between intensity and transition frequency becomes nonlinear. As seen in Fig. 4, the value of the transition wavenumber extrapolated to zero intensity is $13612.37 \mathrm{~cm}^{-1}$. Considering that in this transition the $\mid \begin{array}{lllll}X & 0 & 0\end{array}$ and $\left|\begin{array}{lll}X & 11 & 0\end{array}\right\rangle$ states belong to the $n$ and $n-1$ Fourier manifolds (see Fig. 1), the transition wavenumber between $|X 00\rangle$ and $|X 110\rangle$ can be obtained as $(-13612.37+15220.70)=1608.33 \mathrm{~cm}^{-1}$, where $15220.70 \mathrm{~cm}^{-1}$ is the photon energy of the dressing light. The numerical value for the transition wavenumber, obtained as the difference between the computed field-free eigenenergies of $|X 00\rangle$ and $|X 110\rangle$, is also $1608.33 \mathrm{~cm}^{-1}$; thus, the extrapolation technique works perfectly.

Of course there are spectroscopic methods already available, such as laser induced disperse fluoroesence and stimulated emission pumping [46], capable of producing data similar to those retrieved from the normally forbidden transitions measured by our extrapolation scheme. Nonetheless, light-dressed spectroscopy could complement these existing emission-only spectroscopic methods and it is worth noting that the extrapolation scheme can be utilized in both absorption and emission measurements, and varying the dressinglight wavelength could provide control and selectivity over the transitions to be measured (see below).

\section{Frequency dependence of the light-dressed spectrum}

Figure 5 shows the light-dressed spectrum of $\mathrm{Na}_{2}$ when dressed by $I=10^{8} \mathrm{~W} \mathrm{~cm}^{-2}$ intensity light fields of different wavelength. As seen in Fig. 5, both the absorption and the stimulated emission spectra vary strongly with the dressinglight wavelength. This is expected because with varying dressing-light wavelength the contributions of different fieldfree states in the light-dressed states also vary, leading to varying transition probabilities. Therefore, by changing the dressing-light wavelength, one has a certain control over which types of transitions appear in the light-dressed spectrum. In the vicinity of dressing-light wavelengths which are resonant with a $\left|\begin{array}{lll}A & v & 1\end{array}\right\rangle \leftarrow\left|\begin{array}{lll}X & 0 & 0\end{array}\right\rangle$-type transition, the absorption spectrum signal decreases (vertical white lines in the left panel of Fig. 5) while stimulated emission increases simultaneously. This can be understood as resulting from the increased mixing of field-free states near resonance; i.e., the
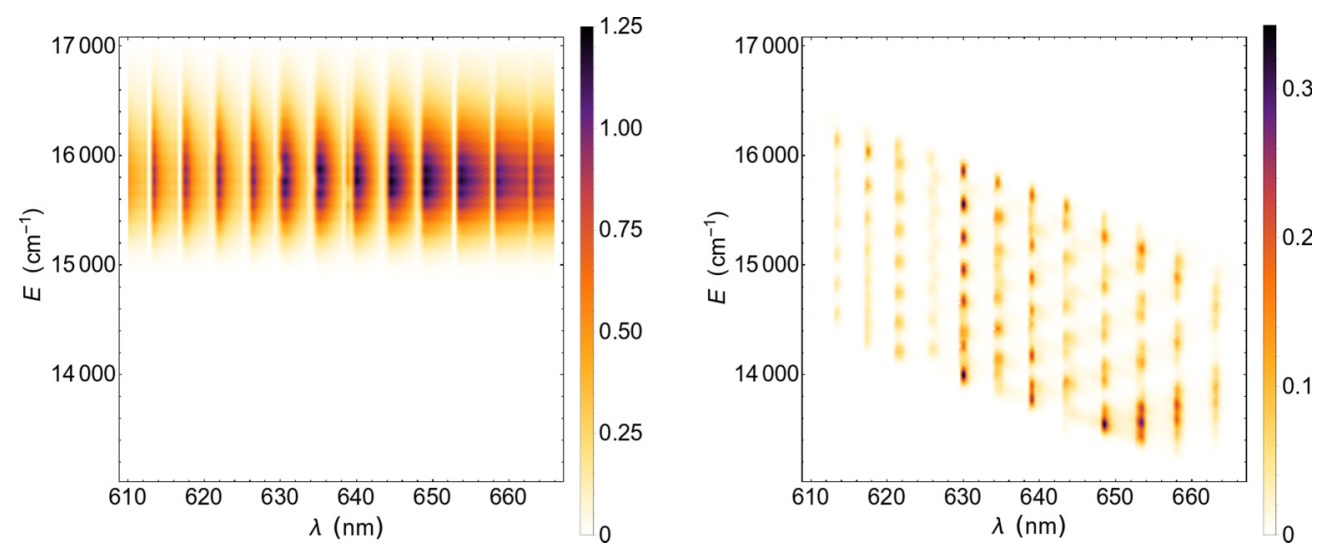

FIG. 5. Absorption (left panel) and stimulated emission (right panel) 0 -K spectra of $\mathrm{Na}_{2}$ dressed with an $I=10^{8} \mathrm{~W} \mathrm{~cm}^{-2}$ intensity laser light of different wavelengths. 

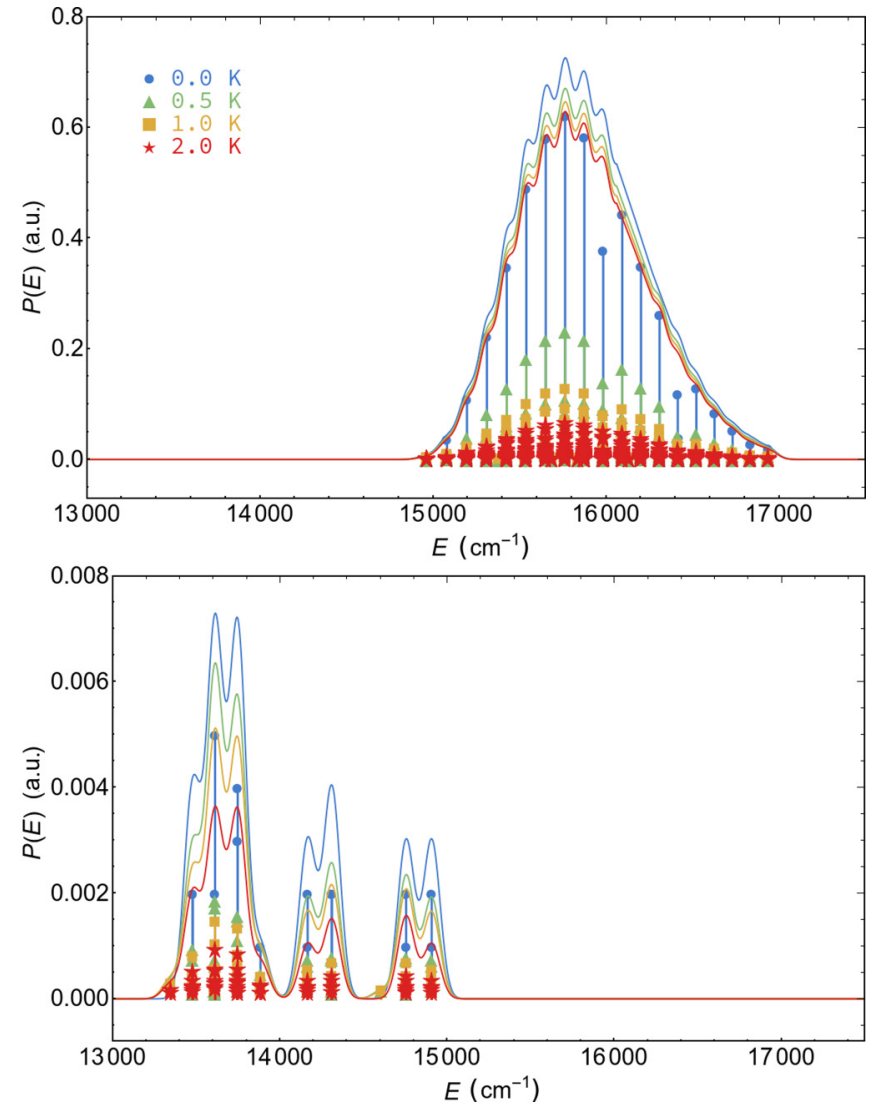

FIG. 6. Light-dressed absorption (upper panel) and stimulated emission (lower panel) spectra of $\mathrm{Na}_{2}$ obtained at different temperatures with a $657 \mathrm{~nm}$ wavelength dressing field having $0.05 \mathrm{GW} \mathrm{cm}^{-2}$ intensity.

weight of $\left|\begin{array}{lll}X & 0 & 0\end{array}\right\rangle$ decreases (leading to a decrease in the absorption), while the weight of $|A v 1\rangle$ increases (leading to an increase in the emission) in the light-dressed state correlating to $\left|\begin{array}{lll}X & 0 & 0\end{array}\right\rangle$.

Interestingly, due to the values of the Frank-Condon overlaps between the vibrational states of the $X$ and $A$ electronic states of $\mathrm{Na}_{2}$, the number of vertical nodes in the stimulated emission spectrum at different dressing-light wavelengths can in some cases reveal which $v$ value of the $|A v J\rangle$-type states contributes the most to the initial light-dressed state. For example, using $662 \mathrm{~nm}$ dressing light leads to emission lines the transition amplitudes of which primarily originate from $|X v J \pm 1\rangle \leftarrow|A 1 J\rangle$-type transitions, while using $657 \mathrm{~nm}$ dressing light leads to emission lines the transition amplitudes of which primarily originate from $|X v J \pm 1\rangle \leftarrow|A 2 J\rangle$-type transitions.

\section{Light-dressed spectra at finite temperatures}

Up to this point the light-dressed spectra shown correspond to $T=0 \mathrm{~K}$; that is, it was assumed that the initial lightdressed state correlates to the field-free rovibronic ground state of $\mathrm{Na}_{2}$. The physical picture behind this assumption is as follows: initially the field-free molecules are all in their ground state and these are changed into dressed states with the adiabatic turn-on of the dressing field. In a realistic
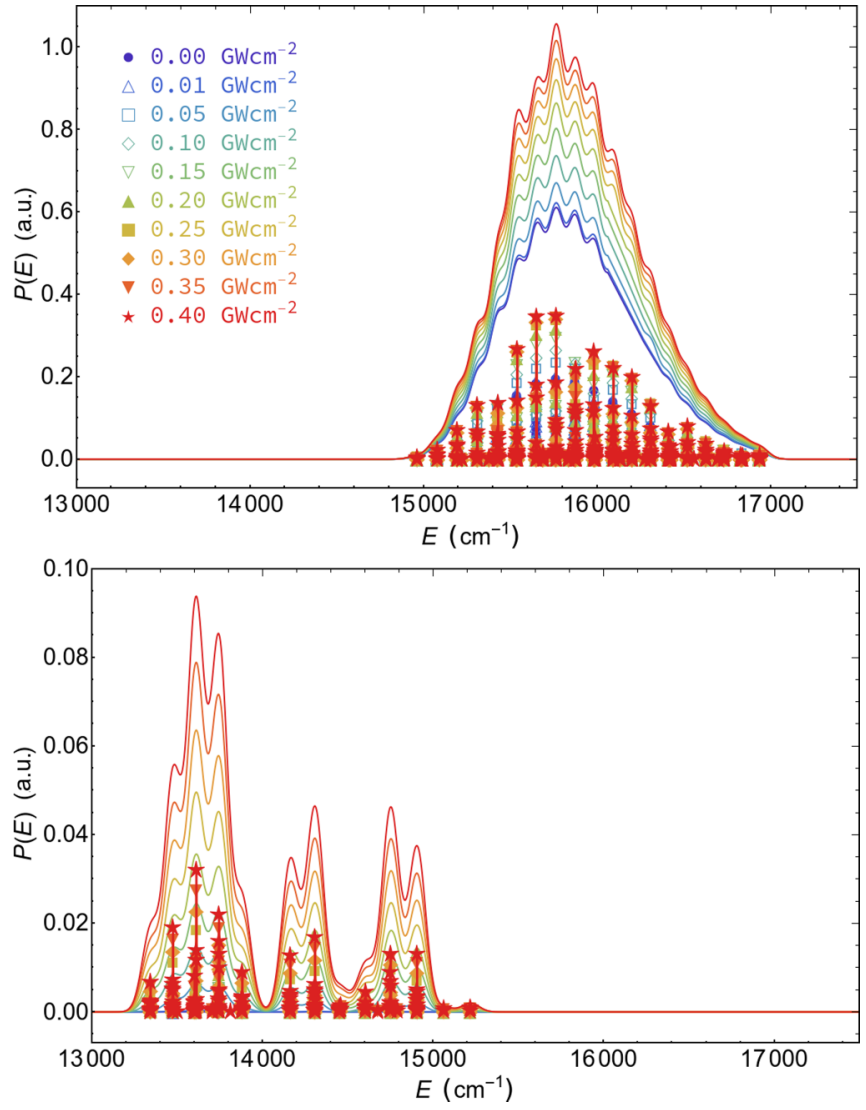

FIG. 7. Light-dressed absorption (upper panel) and stimulated emission (lower panel) spectra of $\mathrm{Na}_{2}$ obtained at $0.5 \mathrm{~K}$ with 657 $\mathrm{nm}$ wavelength dressing fields having different intensities.

experiment at a finite temperature, however, not all the molecules are necessarily in their ground state. Thus, thermal averaging of the computed spectrum needs to be carried out. Since it is assumed that thermal averaging occurs only prior to but not after the light-dressing process, the thermal averaging can be done by weighting transitions with the Boltzmann weights of the field-free states correlating to the respective initial light-dressed states. That is, transitions from each $\left|\Phi_{i}\right\rangle$ light-dressed state are considered in the computed spectrum, but with all transitions from a given $\left|\Phi_{i}\right\rangle$ light-dressed state weighted by

$$
\frac{e^{-E_{i}^{\mathrm{FF}} / k T}}{Q(T)}
$$

where $Q(T)=\sum_{i} e^{-E_{i}^{\mathrm{FF}} / k T}$ is the rovibronic partition function of the field-free molecule and $E_{i}^{\mathrm{FF}}$ is the energy of the field-free rovibronic state to which $\left|\Phi_{i}\right\rangle$ correlates in the limit of the dressing-light intensity going to zero. An additional complication at finite temperatures is that one needs to take into account that the field-free rotational states of a closed-shell diatomic molecule are characterized not only by $J$ but also by the $m$ quantum number, which stands for the projection of the rotational angular momentum onto the chosen space-fixed quantization axis. Since a linearly 

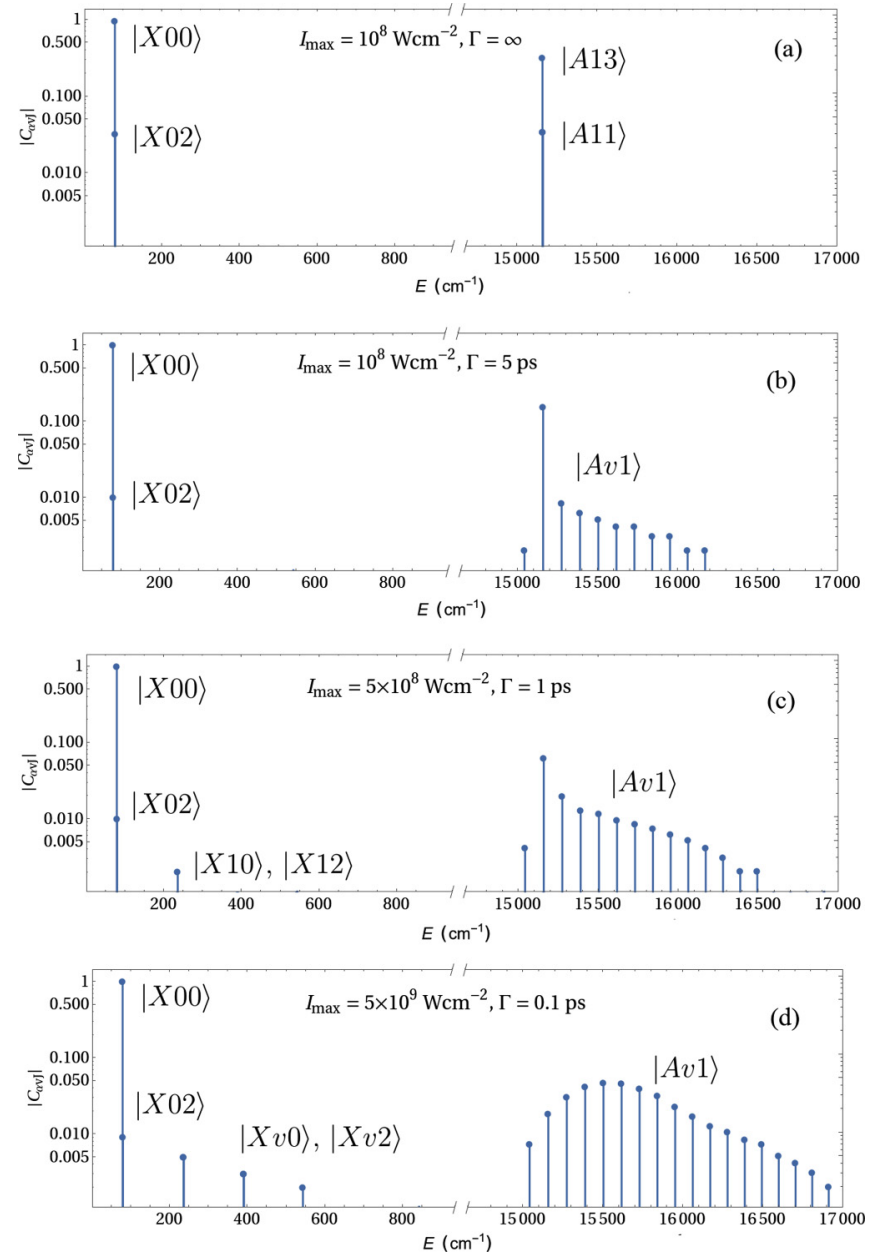

FIG. 8. Population of the different field-free eigenstates in the wave function for $\lambda=663 \mathrm{~nm}$ wavelength dressing fields of different turn-on time and intensity.

polarized dressing field (and the weak probe pulse with identical polarization) cannot mix states with different $m$ quantum numbers, one can simply work in the $m=0$ manifold when $T=0 \mathrm{~K}$. For finite-temperature calculations, however, one needs to determine the light-dressed states and corresponding transitions for the different $m$ manifolds and include them into the spectrum with the appropriate weights shown in Eq. (4).

Figure 6 shows the light-dressed spectra of $\mathrm{Na}_{2}$ at different temperatures, when $\mathrm{Na}_{2}$ is dressed by a $657 \mathrm{~nm}$ wavelength light field of $0.05 \mathrm{GW} \mathrm{cm}^{-2}$ intensity. The upper panel of Fig. 6 demonstrates that although absorption peaks split and peak heights of individual lines decrease significantly with increasing temperature (due to low-lying rotational states being populated at finite temperature) the envelope of the spectrum changes to a much smaller extent. As for stimulated emission, increasing the temperature seems to have a more pronounced effect on the spectrum envelope than in the case of absorption (see the lower panel of Fig. 6).

Figure 7 shows the same dressing-light intensity dependence of the light-dressed spectra as in Fig. 2, but at $T=$ $0.5 \mathrm{~K}$. The same conclusions apply as for Fig. 6; the number of individual lines and the peak amplitudes are much more affected by temperature than the spectrum envelopes.
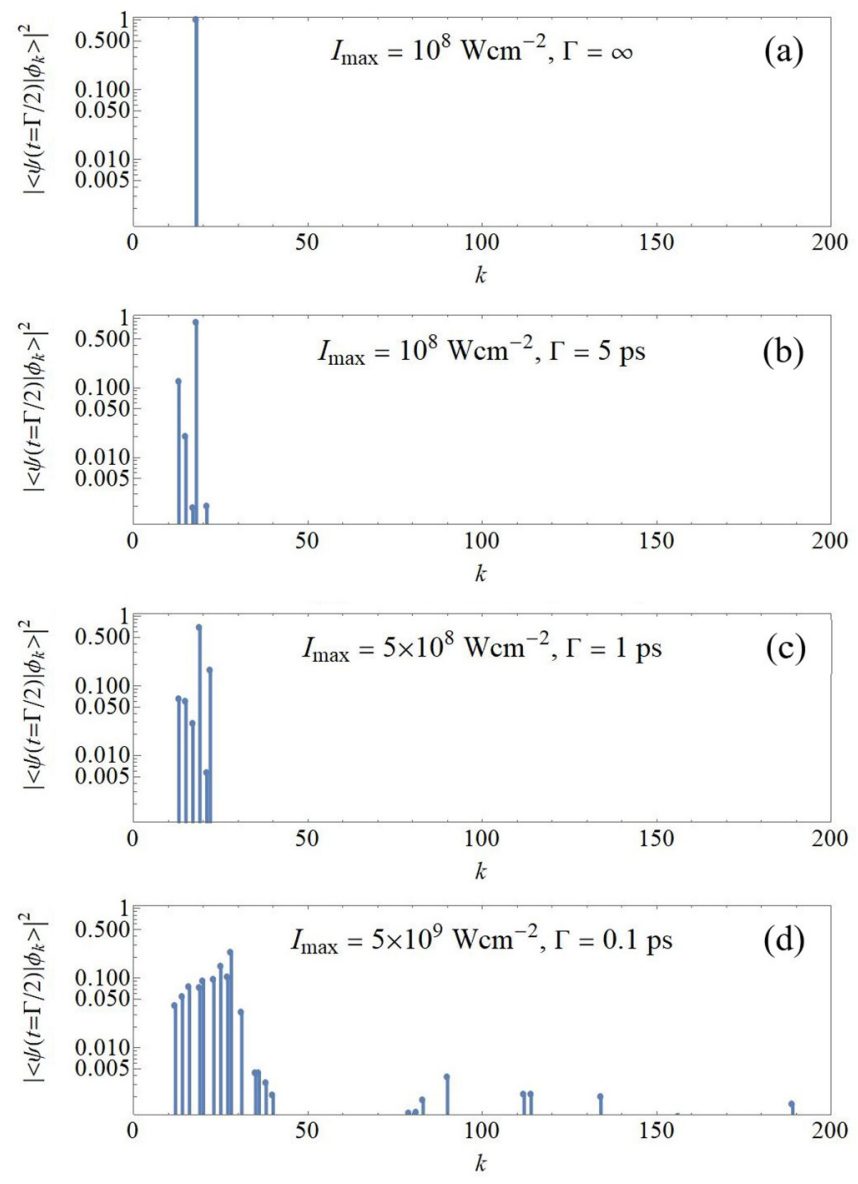

FIG. 9. Population of the different light-dressed states in the wave function for $\lambda=663 \mathrm{~nm}$ wavelength dressing fields of different turn-on time and intensity.

\section{E. Effects of the dressing-field turn-on time on the light-dressed states}

Up to this point it was assumed that the dressing field is turned on adiabatically. As a consequence, starting from an initial field-free state the generated light-dressed wave function is composed of a single light-dressed state, correlating to the initial field-free state. If the dressing field is not turned on adiabatically, then the generated light-dressed wave function becomes a superposition of light-dressed states, with coefficients depending on the turn-on time. Interested readers can find further information and an in-depth investigation on the temporal evolution of light-dressed states in the Floquet formalism, for example, in Refs. [39,40,47].

Figure 8 demonstrates the population of the different fieldfree eigenstates in the wave function for dressing fields of different turn-on time $(\Gamma)$ and intensity. The functional form of the dressing light was assumed to be $\boldsymbol{E}_{1}(t)=\mathbf{0}$ for $t<$ $0, \boldsymbol{E}_{1}(t)=\boldsymbol{E}_{\max } \sin \left(\hbar \omega_{1} t\right) \sin ^{2}(\pi t / \Gamma)$ for $0<t<\Gamma / 2$, and $\boldsymbol{E}_{1}(t)=\boldsymbol{E}_{\max }$ for $\Gamma / 2<t$. The populations shown in Fig. 8 were computed for $t=\Gamma / 2$ by solving the TDSE directly.

Panels (b)-(d) of Fig. 8 demonstrate that when the turn-on time $(\Gamma)$ is shorter than the characteristic timescale of molecular rotations (based on the $\left|\begin{array}{lll}X & 0 & 0\end{array}\right\rangle \leftrightarrow\left|\begin{array}{lll}X & 0 & 1\end{array}\right\rangle$ transition of $\mathrm{Na}_{2}$, this is around $100 \mathrm{ps}$ ) the degree of rotational excitation 
is reduced and in fact in the excited electronic state it is limited to that required by the optical selection rules $(\Delta J= \pm 1)$. Panels (c) and (d) of Fig. 8 also demonstrate that as the dressing pulse becomes shorter in the time domain it expands in the frequency domain, giving rise to significant populations in $|X v 0\rangle$ - and $|A v 1\rangle$-type field-free eigenstates in a wider energy range.

After the dressing field reaches its peak intensity at $t=$ $\Gamma / 2$ (and is kept constant thereafter) the wave function can be expanded as a superposition of the light-dressed states, and the light-dressed spectrum can be calculated using Eq. (C14). Figure 9 depicts the population of the different light-dressed states in the wave function for dressing fields of different turn-on time $(\Gamma)$ and intensity. Figure 9 demonstrates that by decreasing the turn-on time of the dressing field the initial wave function becomes a more and more pronounced mixture of light-dressed states. Nonetheless, if the probe pulse is long enough to average out interferences in the transition probability, then the light-dressed spectrum can be generated as a simple weighted sum of the spectra of individual light-dressed states.

To summarize, by changing the turn-on time of the dressing field, the initial wave function, and thus the transition peaks observed in the light-dressed spectrum, can be influenced or controlled, although this might complicate the interpretation of the spectrum significantly.

\section{SUMMARY AND CONCLUSIONS}

We presented a theoretical method to compute lightdressed spectra of molecules, dressed by medium-intensity classical light fields. Light-dressed spectroscopy is not only of fundamental interest but can also be a useful practical tool for providing valuable spectroscopic data and related insight for both light-dressed and field-free molecular systems. Our approach is based on Floquet theory for deriving the lightdressed states, which are expanded in the basis of fieldfree molecular eigenstates. Once the light-dressed states are determined, transition amplitudes between them, as recorded by a weak probe pulse, are computed using perturbation theory.

Numerical applications are given for the homonuclear diatomic molecule $\mathrm{Na}_{2}$, for which the general formulas can be simplified and the physical processes leading to its rovibronic light-dressed spectra can be understood.

It was found that with respect to the field-free spectrum the light-dressing process leads to the splitting of existing peaks as well as the appearance of new peaks, forbidden in the field-free case. Processes similar in spirit to the well-known Autler-Townes and intensity borrowing effects of molecular spectroscopy could be identified.

The dependence of the light-dressed rovibronic spectrum of $\mathrm{Na}_{2}$ on the dressing-light intensity and the dressing-light wavelength was also investigated. It was found that by manipulating the intensity and the wavelength of the dressing light one can influence, and to some extent control, the peaks appearing in the light-dressed spectra.

We showed that the frequencies of forbidden field-free transitions can be determined by extrapolating light-dressed transition frequencies to the limit of zero dressing-light intensity, and the extrapolation scheme appears to produce excellent results.

Finite temperature calculations, assuming different initial temperatures of the molecular ensemble prior to the dressing process, revealed that the individual field-dressed spectral peaks are much more sensitive to the initial temperature than their envelope, similar to the field-free case.

Finally, it was shown that by changing the turn-on time of the dressing field from the adiabatic limit to shorter times the initial wave function of the light-dressed system can be modified significantly, which is likely to result in changes in the light-dressed spectrum, as well.

\section{ACKNOWLEDGMENTS}

This research was supported by the European Union funded Hungarian Grant No. EFOP-3.6.2-16-2017-00005. The authors are grateful to the Hungarian National Research, Development and Innovation Office (NKFIH) for support (Grants No. PD124623 and No. K119658).

\section{APPENDIX A: DETERMINATION OF LIGHT-DRESSED STATES}

\section{General considerations}

In this section we summarize some aspects of the Floquet approach $[14,37,38]$ used to compute the light-dressed states generated by a $\hat{W}_{1}(t)$ interaction between the molecule and the dressing field. For a Hamiltonian periodic in time, such as

$$
\begin{gathered}
\hat{H}_{d}(t)=\hat{H}_{\mathrm{mol}}+\hat{W}_{1}(t), \\
\hat{H}_{d}(t+T)=\hat{H}_{d}(t),
\end{gathered}
$$

where $T=2 \pi / \omega_{1}$, the TDSE

$$
i \hbar \partial_{t}|\psi(t)\rangle=\hat{H}_{d}(t)|\psi(t)\rangle
$$

has the general solution of the form

$$
|\psi(t)\rangle=\sum_{k} c_{k} e^{-\frac{i}{\hbar} \varepsilon_{k} t}\left|\Phi_{k}(t)\right\rangle,
$$

where $\varepsilon_{k}$ are the so-called quasienergies and the $\left|\Phi_{k}(t)\right\rangle$ Floquet states (also termed light-dressed states in our paper) satisfy

$$
\left|\Phi_{k}(t+T)\right\rangle=\left|\Phi_{k}(t)\right\rangle,
$$

and

$$
\left[\hat{H}_{d}(t)-i \hbar \partial_{t}\right]\left|\Phi_{k}(t)\right\rangle=\hat{H}_{F}(t)\left|\Phi_{k}(t)\right\rangle=\varepsilon_{k}\left|\Phi_{k}(t)\right\rangle .
$$

As can be verified using Eq. (A6), if $\varepsilon_{k}$ is a quasienergy, then $\varepsilon_{k}+\hbar m \omega_{1}$ is also a quasienergy with a corresponding Floquet state $e^{i m \omega_{1} t}\left|\Phi_{k}(t)\right\rangle$. However, it is clear from Eq. (A4) that such a shifted quasienergy does not represent a new physical state, because $\varepsilon_{k}+\hbar m \omega_{1}$ with $e^{i m \omega_{1} t}\left|\Phi_{k}(t)\right\rangle$ give the same contribution to the wave function as $\varepsilon_{k}$ with $\left|\Phi_{k}(t)\right\rangle$.

Because $\left|\Phi_{k}(t)\right\rangle$ are periodic in time, they can be expanded as a Fourier series:

$$
\left|\Phi_{k}(t)\right\rangle=\sum_{n}\left|\varphi_{k n}\right\rangle e^{i n \omega_{1} t}
$$


Combining Eqs. (A6) and (A7) and assuming $\hat{W}_{1}(t)=-\mathbf{E}_{1} \hat{\boldsymbol{\mu}} \cos \left(\omega_{1} t\right)=-\frac{1}{2} \mathbf{E}_{1} \hat{\boldsymbol{\mu}}\left(e^{i \omega_{1} t}+e^{-i \omega_{1} t}\right)$ gives

$$
\left(\hat{H}_{\mathrm{mol}}+\hbar n \omega_{1}\right) \sum_{n}\left|\varphi_{k n}\right\rangle e^{i n \omega_{1} t}-\frac{1}{2} \mathbf{E}_{1} \hat{\boldsymbol{\mu}} \sum_{n}\left|\varphi_{k n}\right\rangle\left(e^{i(n+1) \omega_{1} t}+e^{i(n-1) \omega_{1} t}\right)=\varepsilon_{k} \sum_{n}\left|\varphi_{k n}\right\rangle e^{i n \omega_{1} t} .
$$

Multiplying Eq. (A8) with $\frac{1}{T} e^{-i m \omega_{1} t}$ and integrating on the time period $T$ leads to

$$
\left(\hat{H}_{\mathrm{mol}}+\hbar m \omega_{1}\right)\left|\varphi_{k m}\right\rangle-\frac{1}{2} \mathbf{E}_{1} \hat{\boldsymbol{\mu}}\left(\left|\varphi_{k, m-1}\right\rangle+\left|\varphi_{k, m+1}\right\rangle\right)=\varepsilon_{k}\left|\varphi_{k m}\right\rangle .
$$

The Fourier components $\left|\varphi_{k m}\right\rangle$ can be further expressed as a linear combination of rovibronic molecular states:

$$
\left|\varphi_{k m}\right\rangle=\sum_{\alpha, v, J} C_{m, \alpha v J}^{(k)}|\alpha v J\rangle,
$$

where $\alpha, v$, and $J$ represent electronic, vibrational, and rotational quantum numbers, respectively. Using the expansion of Eq. (A10), Eq. (A9) can be turned into the matrix eigenvalue problem

$$
\sum_{n, \alpha, v, J}\left(\mathbf{H}_{F}\right)_{m \alpha^{\prime} v^{\prime} J^{\prime}, n \alpha v J} C_{n, \alpha v J}^{(k)}=\varepsilon_{k} C_{m, \alpha^{\prime} v^{\prime} J^{\prime}}^{(k)}
$$

where

$$
\left(\mathbf{H}_{F}\right)_{m \alpha^{\prime} v^{\prime} J^{\prime}, n \alpha v J}=\left(\left\langle\alpha^{\prime} v^{\prime} J^{\prime}\left|\hat{H}_{\mathrm{mol}}\right| \alpha v J\right\rangle+\hbar m \omega_{1} \delta_{\alpha \alpha^{\prime}} \delta_{v v^{\prime}} \delta_{J J^{\prime}}\right) \delta_{n m}-\frac{1}{2}\left\langle\alpha^{\prime} v^{\prime} J^{\prime}\left|\mathbf{E}_{1} \hat{\boldsymbol{\mu}}\right| \alpha v J\right\rangle\left(\delta_{n, m-1}+\delta_{n, m+1}\right) .
$$

The pictorial representation of $\mathbf{H}_{F}$ reads

$$
\mathbf{H}_{F}=\left[\begin{array}{cccccccc}
\ddots & \vdots & \vdots & \vdots & \vdots & \vdots & \vdots & \ddots \\
\cdots & \mathbf{H}_{A}+\hbar \omega_{1} \mathbf{I} & \lambda & \mathbf{g}_{A A} & \mathbf{g}_{A X} & 0 & 0 & \cdots \\
\cdots & \lambda^{\dagger} & \mathbf{H}_{X}+\hbar \omega_{1} \mathbf{I} & \mathbf{g}_{X A} & \mathbf{g}_{X X} & 0 & 0 & \cdots \\
\cdots & \mathbf{g}_{A A}^{\dagger} & \mathbf{g}_{X A}^{\dagger} & \mathbf{H}_{A} & \lambda & \mathbf{g}_{A A} & \mathbf{g}_{A X} & \cdots \\
\cdots & \mathbf{g}_{A X}^{\dagger} & \mathbf{g}_{X X}^{\dagger} & \lambda^{\dagger} & \mathbf{H}_{X} & \mathbf{g}_{X A} & \mathbf{g}_{X X} & \cdots \\
\cdots & 0 & 0 & \mathbf{g}_{A A}^{\dagger} & \mathbf{g}_{X A}^{\dagger} & \mathbf{H}_{A}-\hbar \omega_{1} \mathbf{I} & \lambda & \cdots \\
\cdots & 0 & 0 & \mathbf{g}_{A X}^{\dagger} & \mathbf{g}_{X X}^{\dagger} & \lambda^{\dagger} & \mathbf{H}_{X}-\hbar \omega_{1} \mathbf{I} & \cdots \\
\therefore & \vdots & \vdots & \vdots & \vdots & \vdots & \vdots & \ddots
\end{array}\right],
$$

where different matrix elements represent different values of the $\alpha$ electronic and $n$ Fourier indices of Eq. (A12) (for the sake of simplicity, we assumed only two electronic states, labeled $X$ and $A$ ), and each matrix element in Eq. (A13) is itself a matrix representation of different operators in the space of rovibrational states, i.e.,

$$
\begin{gathered}
\left(\mathbf{H}_{A}\right)_{v^{\prime} J^{\prime}, v J}=\left\langle A v^{\prime} J^{\prime}\left|\hat{H}_{\mathrm{mol}}\right| A v J\right\rangle, \\
\left(\mathbf{H}_{X}\right)_{v^{\prime} J^{\prime}, v J}=\left\langle X v^{\prime} J^{\prime}\left|\hat{H}_{\mathrm{mol}}\right| X v J\right\rangle, \\
(\boldsymbol{\lambda})_{v^{\prime} J^{\prime}, v J}=\left\langle A v^{\prime} J^{\prime}\left|\hat{H}_{\mathrm{mol}}\right| X v J\right\rangle, \\
\left(\mathbf{g}_{\alpha \beta}\right)_{v^{\prime} J^{\prime}, v J}=-\frac{1}{2}\left\langle\alpha v^{\prime} J^{\prime}\left|\mathbf{E}_{1} \hat{\boldsymbol{\mu}}\right| \beta v J\right\rangle,
\end{gathered}
$$

and $\mathbf{I}$ is the identity matrix. $\mathbf{H}_{A}$ and $\mathbf{H}_{X}$ can be thought of as the rovibrational Hamiltonians in the adiabatic electronic states $A$ and $X$, respectively, while $\lambda$ accounts for intrinsic nonadiabatic couplings between the $X$ and $A$ electronic states. $\mathbf{g}_{\alpha \beta}$ naturally represents the coupling induced by the dressing field.

\section{Simplifying assumptions}

In practical applications, Eq. (A13) can often be simplified.

(1) If intrinsic nonadiabatic couplings can be neglected in the system under investigation, then $\lambda=\mathbf{0}$.

(2) If the molecule has no permanent dipole, then $\mathbf{g}_{X X}=$ $\mathbf{g}_{A A}=\mathbf{0}$.

(3) Finally, if $\hbar \omega_{1}$ is resonant with the electronic excitation between the states $X$ and $A$, then nonresonant coupling terms can be neglected up to moderate field strengths [41]. This leaves only those $\mathbf{g}_{\alpha \beta}$ matrices nonzero which connect $\mathbf{H}_{X}+$ $n \hbar \omega_{1} \mathbf{I}$ - and $\mathbf{H}_{A}+(n-1) \hbar \omega_{1} \mathbf{I}$-type elements.

With the above three simplifications, $\mathbf{H}_{F}$ becomes block diagonal with each $2 \times 2$ block being identical up to a constant shift. The block labeled with the Fourier index $n$ reads

$$
\mathbf{H}_{F}^{2 \times 2}(n)=\left[\begin{array}{cc}
\mathbf{H}_{X}+n \hbar \omega_{1} & \mathbf{g}_{X A} \\
\mathbf{g}_{X A}^{\dagger} & \mathbf{H}_{A}+(n-1) \hbar \omega_{1} \mathbf{I}
\end{array}\right] .
$$

Therefore, instead of solving the general case of Eq. (A9), it becomes sufficient to solve the eigenvalue problem for $\mathbf{H}_{F}^{2 \times 2}(n)$ in order to obtain the light-dressed states and corresponding quasienergies. 


\section{APPENDIX B: TEMPORAL EVOLUTION OF A LIGHT-DRESSED SYSTEM}

In the representation utilized in Eq. (A12) the $\mathbf{H}_{F}$ matrix of the Floquet Hamiltonian and the $C_{n, \alpha v J}^{(k)}$ expansion coefficients of the Floquet states are time independent. Based on this representation and Eqs. (A4) and (A6), the temporal evolution of a light-dressed system can be expressed as [14]

$$
\begin{aligned}
\boldsymbol{\Psi}(t) & =\sum_{k} c_{k} e^{-\frac{i}{\hbar} \mathbf{H}_{F} t} \boldsymbol{\Phi}_{k}=e^{-\frac{i}{\hbar} \mathbf{H}_{F} t} \sum_{k} c_{k} \boldsymbol{\Phi}_{k} \\
& =e^{-\frac{i}{\hbar} \mathbf{H}_{F} t} \boldsymbol{\Psi}(t=0),
\end{aligned}
$$

which is formally equivalent to the temporal evolution of a system with a time-independent Hamiltonian.

In a physical scenario when the dressing-field amplitude is not constant but slowly changes in time, those matrix elements of $\mathbf{H}_{F}$ which represent light-matter couplings also slowly change in time. For a dressing field which is turned on much slower than the characteristic timescales of the field-free system (adiabatically), Eq. (B1) suggests that the well-known adiabatic theorem could be used to predict the temporal changes in $\boldsymbol{\Psi}(t)$. Therefore, in the limit of the dressing-light intensity going to zero $\left(\mathbf{g}_{X A} \rightarrow 0\right)$, the field-free eigenstates are eigenstates of $\mathbf{H}_{F}$, as well; therefore, an adiabatic turn-on of the dressing field will convert an initial field-free eigenstate into a single light-dressed state. This means that light-dressed states and field-free states can be correlated in a one-to-one fashion. However, it is important to mention that the previous two sentences are not true if $\omega_{1}$ is in exact resonance with an allowed transition, because this results in the field-free eigenstates not being eigenstates of $\mathbf{H}_{F}$ (but being a linear combination of $\mathbf{H}_{F}$ eigenstates) even for infinitesimal light-matter coupling strengths. Further information on the temporal evolution of light-dressed states in the Floquet formalism can be found, for example, in Ref. [39], while Refs. [40,47-49] provide examples for the utilization of the adiabatic Floquet dynamics.

\section{APPENDIX C: COMPUTING TRANSITIONS BETWEEN LIGHT-DRESSED STATES}

\section{General considerations}

Let the molecule interact with two periodic electric fields. The full Hamiltonian reads as

$$
\hat{H}(t)=\hat{H}_{\mathrm{mol}}+\hat{W}_{1}(t)+\hat{W}_{2}(t),
$$

where $\hat{H}_{\text {mol }}$ is the field-free molecular Hamiltonian, and $\hat{W}_{1}(t)$ and $\hat{W}_{2}(t)$ account for the interaction between the molecule and the two fields, i.e., in the dipole approximation

$$
\begin{aligned}
& \hat{W}_{1}(t)=-\mathbf{E}_{1} \hat{\boldsymbol{\mu}} \cos \left(\omega_{1} t+\phi\right), \\
& \hat{W}_{2}(t)=-\mathbf{E}_{2} \hat{\boldsymbol{\mu}} \cos \left(\omega_{2} t\right) .
\end{aligned}
$$

$\hat{W}_{1}(t)$ is considered to be generating the light-dressed states, while $\hat{W}_{2}(t)$ originates from a weak probe pulse used to record the spectrum of the light-dressed molecule. The formation of light-dressed states by $\hat{W}_{1}(t)$ is accounted for within the Floquet approach, as described in Appendix A.

For computing the $\hat{W}_{2}(t)$-induced transition amplitudes between the (superposition of) light-dressed states, TDPT1 is used. To derive our working equations, we start with the TDSE containing the interaction with both the dressing and the probe fields:

$$
\begin{aligned}
i \hbar \partial_{t}|\Psi(t)\rangle & =\left[\hat{H}_{\mathrm{mol}}+\hat{W}_{1}(t)+\hat{W}_{2}(t)\right]|\Psi(t)\rangle \\
& =\left[\hat{H}_{d}(t)+\hat{W}_{2}(t)\right]|\Psi(t)\rangle .
\end{aligned}
$$

Equation (C3) is transformed to the interaction picture using the transformation

$$
\left|\Psi_{\mathrm{I}}(t)\right\rangle=e^{\frac{i}{\hbar} \int_{t_{0}}^{t} \hat{H}_{d}\left(t^{\prime}\right) d t^{\prime}}|\Psi(t)\rangle,
$$

which leads to

$$
i \hbar \partial_{t}\left|\Psi_{\mathrm{I}}(t)\right\rangle=\hat{W}_{2 \mathrm{I}}(t)\left|\Psi_{\mathrm{I}}(t)\right\rangle,
$$

where $\hat{W}_{2 \mathrm{I}}(t)=e^{\frac{i}{\hbar} \int_{t_{0}}^{t} \hat{H}_{d}\left(t^{\prime}\right) d t^{\prime}} \hat{W}_{2}(t) e^{-\frac{i}{\hbar} \int_{t_{0}}^{t} \hat{H}_{d}\left(t^{\prime}\right) d t^{\prime}}$. Following the usual TDPT1 procedure of integrating Eq. (C5) from $t_{0}$ to $t$ and applying a successive approximation to express $\left|\Psi_{\mathrm{I}}(t)\right\rangle$ gives

$$
\left|\Psi_{\mathrm{I}}(t)\right\rangle=\hat{U}\left(t, t_{0}\right)\left|\Psi_{\mathrm{I}}\left(t_{0}\right)\right\rangle
$$

with

$$
\begin{aligned}
\hat{U}\left(t, t_{0}\right)=\hat{I} & +\frac{1}{i \hbar} \int_{t_{0}}^{t} \hat{W}_{2 \mathrm{I}}\left(t^{\prime}\right) d t^{\prime} \\
& +\frac{1}{(i \hbar)^{2}} \int_{t_{0}}^{t} \hat{W}_{2 \mathrm{I}}\left(t^{\prime}\right) \int_{t_{0}}^{t^{\prime}} \hat{W}_{2 \mathrm{I}}\left(t^{\prime \prime}\right) d t^{\prime \prime} d t^{\prime}+\cdots .
\end{aligned}
$$

Considering the first two terms of the propagator in Eq. (C7) leads to

$$
\left|\Psi_{\mathrm{I}}(t)\right\rangle=\left|\Psi_{\mathrm{I}}\left(t_{0}\right)\right\rangle+\frac{1}{i \hbar} \int_{t_{0}}^{t} \hat{W}_{2 \mathrm{I}}\left(t^{\prime}\right) d t^{\prime}\left|\Psi_{\mathrm{I}}\left(t_{0}\right)\right\rangle .
$$

The transition amplitude to a final state $\left|\Psi_{\mathrm{I}}^{(F)}\right\rangle$ at time $t$ is thus

$$
\begin{aligned}
\left\langle\Psi_{\mathrm{I}}^{(F)} \mid \Psi_{\mathrm{I}}(t)\right\rangle= & \left\langle\Psi_{\mathrm{I}}^{(F)} \mid \Psi_{\mathrm{I}}\left(t_{0}\right)\right\rangle \\
& +\frac{1}{i \hbar} \int_{t_{0}}^{t}\left\langle\Psi_{\mathrm{I}}^{(F)}\left|\hat{W}_{2 \mathrm{I}}\left(t^{\prime}\right)\right| \Psi_{\mathrm{I}}\left(t_{0}\right)\right\rangle d t^{\prime} .
\end{aligned}
$$

By expanding $\left|\Psi_{\mathrm{I}}^{(F)}\right\rangle$ and $\left|\Psi_{\mathrm{I}}\left(t_{0}\right)\right\rangle$ as a superposition of the $\left|\Phi_{k}\left(t_{0}\right)\right\rangle$ Floquet states, i.e.,

$$
\left|\Psi_{\mathrm{I}}^{(F)}\right\rangle=\sum_{l} a_{l} e^{-\frac{i}{\hbar} \varepsilon t_{0}}\left|\Phi_{l}\left(t_{0}\right)\right\rangle
$$

and

$$
\left|\Psi_{\mathrm{I}}\left(t_{0}\right)\right\rangle=\sum_{k} b_{k} e^{-\frac{i}{\hbar} \varepsilon_{k} t_{0}}\left|\Phi_{k}\left(t_{0}\right)\right\rangle,
$$

Eq. (C9) gives

$$
\left\langle\Psi_{\mathrm{I}}^{(F)} \mid \Psi_{\mathrm{I}}(t)\right\rangle=\sum_{l, k} a_{l}^{*} b_{k} e^{-\frac{i}{\hbar}\left(\varepsilon_{k}-\varepsilon_{l}\right) t_{0}}\left\langle\Phi_{l}\left(t_{0}\right) \mid \Phi_{k}\left(t_{0}\right)\right\rangle+\frac{1}{i \hbar} \sum_{l, k} a_{l}^{*} b_{k} \int_{t_{0}}^{t} e^{-\frac{i}{\hbar}\left(\varepsilon_{k}-\varepsilon_{l}\right) t_{0}}\left\langle\Phi_{l}\left(t_{0}\right)\left|\hat{W}_{2 \mathrm{I}}\left(t^{\prime}\right)\right| \Phi_{k}\left(t_{0}\right)\right\rangle d t^{\prime}
$$




$$
\begin{aligned}
= & \sum_{l, k} a_{l}^{*} b_{k} e^{-\frac{i}{\hbar}\left(\varepsilon_{k}-\varepsilon_{l}\right) t_{0}}\left\langle\Phi_{l}\left(t_{0}\right) \mid \Phi_{k}\left(t_{0}\right)\right\rangle+\frac{1}{i \hbar} \sum_{l, k} a_{l}^{*} b_{k} \int_{t_{0}}^{t} e^{-\frac{i}{\hbar}\left(\varepsilon_{k}-\varepsilon_{l}\right) t_{0}}\left\langle\Phi_{l}\left(t_{0}\right)\right| e^{\frac{i}{\hbar} \int_{t_{0}}^{t^{\prime}} \hat{H}_{d}\left(t^{\prime \prime}\right) d t^{\prime \prime}} \hat{W}_{2}\left(t^{\prime}\right) \\
& \times e^{-\frac{i}{\hbar} \int_{t_{0}}^{t^{\prime}} \hat{H}_{d}\left(t^{\prime \prime}\right) d t^{\prime \prime}}\left|\Phi_{k}\left(t_{0}\right)\right\rangle d t^{\prime} .
\end{aligned}
$$

Because $e^{-\frac{i}{\hbar} \varepsilon_{k} t}\left|\Phi_{k}(t)\right\rangle$ is a solution of the TDSE of Eq. (A3), the effect of the $\hat{U}_{d}\left(t^{\prime}, t_{0}\right)=e^{-\frac{i}{\hbar} t_{t_{0}}^{t^{\prime}} \hat{H}_{d}\left(t^{\prime \prime}\right) d t^{\prime \prime}}$ operator, describing $\hat{H}_{d}(t)$ governed time evolution from $t_{0}$ to $t^{\prime}$, can be evaluated as $\hat{U}_{d}\left(t^{\prime}, t_{0}\right)\left[e^{-\frac{i}{\hbar} \varepsilon_{k} t_{0}}\left|\Phi_{k}\left(t_{0}\right)\right\rangle\right]=e^{-\frac{i}{\hbar} \varepsilon_{k} t^{\prime}}\left|\Phi_{k}\left(t^{\prime}\right)\right\rangle$. By exploiting this fact, one arrives at

$$
\left\langle\Psi_{\mathrm{I}}^{(F)} \mid \Psi_{\mathrm{I}}(t)\right\rangle=\sum_{l, k} a_{l}^{*} b_{k} e^{-\frac{i}{\hbar}\left(\varepsilon_{k}-\varepsilon_{l}\right) t_{0}}\left\langle\Phi_{l}\left(t_{0}\right) \mid \Phi_{k}\left(t_{0}\right)\right\rangle+\frac{1}{i \hbar} \sum_{l, k} a_{l}^{*} b_{k} \int_{t_{0}}^{t}\left\langle\Phi_{l}\left(t^{\prime}\right)\left|\hat{W}_{2}\left(t^{\prime}\right)\right| \Phi_{k}\left(t^{\prime}\right)\right\rangle e^{-\frac{i}{\hbar}\left(\varepsilon_{k}-\varepsilon_{l}\right) t^{\prime}} d t^{\prime} .
$$

Finally, using the explicit form of $\hat{W}_{2}(t)$ given in Eq. (C2) and expressing the periodic $\left|\Phi_{k}(t)\right\rangle$ functions under the integral with their Fourier series [see Eq. (A7)] leads to

$$
\left\langle\Psi_{\mathrm{I}}^{(F)} \mid \Psi_{\mathrm{I}}(t)\right\rangle=\sum_{l, k} a_{l}^{*} b_{k} e^{-\frac{i}{\hbar}\left(\varepsilon_{k}-\varepsilon_{l}\right) t_{0}}\left\langle\Phi_{l}\left(t_{0}\right) \mid \Phi_{k}\left(t_{0}\right)\right\rangle-\frac{1}{2 i \hbar} \sum_{l, k} a_{l}^{*} b_{k} \sum_{n, m} \int_{t_{0}}^{t}\left\langle\varphi_{l n}\left|\mathbf{E}_{2} \hat{\boldsymbol{\mu}}\right| \varphi_{k m}\right\rangle e^{-\frac{i}{\hbar}\left(\hbar \omega_{1}(n-m)+\varepsilon_{k}-\varepsilon_{l} \pm \hbar \omega_{2}\right) t^{\prime}} d t^{\prime}
$$

\section{Molecules with no permanent dipole}

For molecules with no permanent dipole and negligible intrinsic nonadiabatic couplings, the light-dressed states determined within a Floquet approach, in which nonresonant coupling terms with the dressing field are neglected [see Eqs. (A18) and (A7)], can be written as

$$
\left|\Phi_{k}(t)\right\rangle=\sum_{n}\left(\left|\alpha_{k n}\right\rangle+\left|\beta_{k(n-1)}\right\rangle e^{-i \omega_{1} t}\right) e^{i n \omega_{1} t},
$$

where $\left|\alpha_{k n}\right\rangle$ and $\left|\beta_{k(n-1)}\right\rangle$ represent the two manifolds of rovibronic states with Fourier indices $n$ and $n-1$, respectively. Because neglecting the nonresonant coupling terms in the Floquet approach means that the Floquet Hamiltonian becomes block diagonal [see Eq. (A18)], and that $\left|\alpha_{k n}\right\rangle$ and $\left|\beta_{k(n-1)}\right\rangle$ become the same for all $n$, Eq. (C15) simplifies to

$$
\left|\Phi_{k}(t)\right\rangle=\left(\left|\alpha_{k}\right\rangle+\left|\beta_{k}\right\rangle e^{-i \omega_{1} t}\right) e^{i n \omega_{1} t},
$$

where $\left|\Phi_{k}(t)\right\rangle$ is a Floquet state obtained from the $n$th $2 \times$ 2 block of the Floquet Hamiltonian, and the quasienergy corresponding to $\left|\Phi_{k}(t)\right\rangle$ may be written as $\varepsilon_{k}+\hbar n \omega_{1}$. As explained under Eq. (A6), if the quasienergy is shifted by $-\hbar n \omega_{1}$ and the Floquet state is multiplied with $e^{-i n \omega_{1} t}$ one arrives at an equivalent physical state. Therefore, Eq. (C16) can be rewritten as

$$
\left|\Phi_{k}(t)\right\rangle=\left|\alpha_{k}\right\rangle+\left|\beta_{k}\right\rangle e^{-i \omega_{1} t}
$$

with the corresponding quasienergy of $\varepsilon_{k}$. Using Eq. (C17) instead of Eq. (A7) leads to a simplified version of Eq. (C14), that is

$$
\begin{aligned}
\left\langle\Psi_{\mathrm{I}}^{(F)}\right. & \left|\Psi_{\mathrm{I}}(t)\right\rangle \\
= & \sum_{l, k} a_{l}^{*} b_{k} e^{-\frac{i}{\hbar}\left(\varepsilon_{k}-\varepsilon_{l}\right) t_{0}}\left\langle\Phi_{l}\left(t_{0}\right) \mid \Phi_{k}\left(t_{0}\right)\right\rangle \\
& -\frac{1}{2 i \hbar} \sum_{l, k} a_{l}^{*} b_{k} \int_{t_{0}}^{t}\left\langle\beta_{l}\left|\mathbf{E}_{2} \hat{\boldsymbol{\mu}}\right| \alpha_{k}\right\rangle e^{-\frac{i}{\hbar}\left(\varepsilon_{k}-\varepsilon_{l}-\hbar \omega_{1} \pm \hbar \omega_{2}\right) t^{\prime}} d t^{\prime} \\
& -\frac{1}{2 i \hbar} \sum_{l, k} a_{l}^{*} b_{k} \int_{t_{0}}^{t}\left\langle\alpha_{l}\left|\mathbf{E}_{2} \hat{\boldsymbol{\mu}}\right| \beta_{k}\right\rangle e^{-\frac{i}{\hbar}\left(\varepsilon_{k}-\varepsilon_{l}+\hbar \omega_{1} \pm \hbar \omega_{2}\right) t^{\prime}} d t^{\prime},
\end{aligned}
$$

where we exploited the fact that $\left\langle\alpha_{l}\left|\mathbf{E}_{2} \hat{\boldsymbol{\mu}}\right| \alpha_{k}\right\rangle=$ $\left\langle\beta_{l}\left|\mathbf{E}_{2} \hat{\boldsymbol{\mu}}\right| \beta_{k}\right\rangle=0$ for a molecule with no permanent dipole. Continuing with the standard TDPT1 procedure, the second and third terms in Eq. (C18) lead to the two terms of Eq. (3).
[1] F. Merkt and M. Quack, in Handbook of High-Resolution Spectroscopy, edited by M. Quack and F. Merkt (Wiley, New York, 2011).

[2] T. Udem, R. Holzwarth, and T. W. Hänsch, Nature (London) 416, 233 (2002).

[3] S. A. Diddams, J. Opt. Soc. Am. B 27, B51 (2010).

[4] J. L. Hall, Rev. Mod. Phys. 78, 1279 (2006).

[5] T. W. Hänsch, Rev. Mod. Phys. 78, 1297 (2006).

[6] T. Brabec and F. Krausz, Rev. Mod. Phys. 72, 545 (2000).

[7] F. Krausz and M. Ivanov, Rev. Mod. Phys. 81, 163 (2009).

[8] A. Palacios and F. Martín, WIREs Comp. Mol. Sci. e1430 (2019).
[9] A. H. Zewail, J. Phys. Chem. A 104, 5660 (2000).

[10] M. Dantus, Annu. Rev. Phys. Chem. 52, 639 (2001).

[11] S. Mukamel, Annu. Rev. Phys. Chem. 51, 691 (2000).

[12] T. Ando, A. Iwasaki, and K. Yamanouchi, Phys. Rev. Lett. 120, 263002 (2018).

[13] C. Cohen-Tannoudji, J. Dupont-Roc, and G. Grynberg, AtomPhoton Interactions: Basic Processes and Applications (Wiley, New York, 2004).

[14] J. H. Shirley, Phys. Rev. 138, B979 (1965).

[15] T. F. George, I. H. Zimmerman, J.-M. Yuan, J. R. Laing, and P. L. DeVries, Acc. Chem. Res. 10, 449 (1977).

[16] C. Wunderlich, E. Kobler, H. Figger, and T. W. Hänsch, Phys. Rev. Lett. 78, 2333 (1997). 
[17] B. M. Garraway and K.-A. Suominen, Phys. Rev. Lett. 80, 932 (1998).

[18] Y. Sato, H. Kono, S. Koseki, and Y. Fujimura, J. Am. Chem. Soc. 125, 8019 (2003).

[19] M. E. Corrales, J. González-Vázquez, G. Balerdi, I. R. Solá, R. de Nalda, and L. Bañares, Nat. Chem. 6, 785 (2014).

[20] F. Kelkensberg, C. Lefebvre, W. Siu, O. Ghafur, T. T. NguyenDang, O. Atabek, A. Keller, V. Serov, P. Johnsson, M. Swoboda, T. Remetter, A. L'Huillier, S. Zherebtsov, G. Sansone, E. Benedetti, F. Ferrari, M. Nisoli, F. Lépine, M. F. Kling, and M. J. J. Vrakking, Phys. Rev. Lett. 103, 123005 (2009).

[21] O. Atabek, R. Lefebvre, and T. Nguyen-Dang, in Unstable States in the Continuous Spectra, Part I: Analysis, Concepts, Methods, and Results (Elsevier, New York, 2010), pp. 51-104.

[22] P. H. Bucksbaum, A. Zavriyev, H. G. Muller, and D. W. Schumacher, Phys. Rev. Lett. 64, 1883 (1990).

[23] S. H. Autler and C. H. Townes, Phys. Rev. 100, 703 (1955).

[24] A. Sanli, X. Pan, S. Magnier, J. Huennekens, A. M. Lyyra, and E. H. Ahmed, J. Chem. Phys. 147, 204301 (2017).

[25] E. H. Ahmed, J. Huennekens, T. Kirova, J. Qi, and A. M. Lyyra, Adv. At. Mol. Opt. Phys. 61, 467 (2012).

[26] C. Y. Lee and B. H. Pate, J. Chem. Phys. 107, 10430 (1997).

[27] B. Gu and I. Franco, Phys. Rev. A 98, 063412 (2018).

[28] G. J. Halász, A. Vibók, M. Šindelka, N. Moiseyev, and L. S. Cederbaum, J. Phys. B 44, 175102 (2011).

[29] G. J. Halász, M. Šindelka, N. Moiseyev, L. S. Cederbaum, and A. Vibók, J. Phys. Chem. A 116, 2636 (2012).

[30] T. Szidarovszky, G. J. Halász, A. G. Császár, L. S. Cederbaum, and Á. Vibók, J. Phys. Chem. Lett. 9, 2739 (2018).

[31] T. Szidarovszky, G. J. Halász, A. G. Császár, L. S. Cederbaum, and Á. Vibók, J. Phys. Chem. Lett. 9, 6215 (2018).

[32] W. S. Warren, H. Rabitz, and M. Dahleh, Science 259, 1581 (1993).
[33] C. P. Koch and M. Shapiro, Chem. Rev. 112, 4928 (2012).

[34] N. V. Vitanov, A. A. Rangelov, B. W. Shore, and K. Bergmann, Rev. Mod. Phys. 89, 015006 (2017).

[35] O. Atabek, R. Lefebvre, M. Lepers, A. Jaouadi, O. Dulieu, and V. Kokoouline, Phys. Rev. Lett. 106, 173002 (2011).

[36] M. Viteau, A. Chotia, M. Allegrini, N. Bouloufa, O. Dulieu, D. Comparat, and P. Pillet, Science 321, 232 (2008).

[37] S.-I. Chu and D. A. Telnov, Phys. Rep. 390, 1 (2004).

[38] S.-I. Chu, in Advances in Atomic and Molecular Physics (Elsevier, New York, 1985), pp. 197-253.

[39] S. Guérin and H. R. Jauslin, in Advances in Chemical Physics (Wiley, New York, 2003), pp. 147-267.

[40] C. Lefebvre, T. T. Nguyen-Dang, F. Dion, M. J. J. Vrakking, V. N. Serov, and O. Atabek, Phys. Rev. A 88, 053416 (2013).

[41] G. J. Halász, Á. Vibók, N. Moiseyev, and L. S. Cederbaum, J. Phys. B 45, 135101 (2012).

[42] P. R. Bunker and P. Jensen, Molecular Symmetry and Spectroscopy (NRC, Ottawa, 1998).

[43] S. Magnier, P. Millié, O. Dulieu, and F. Masnou-Seeuws, J. Chem. Phys. 98, 7113 (1993).

[44] W. T. Zemke, K. K. Verma, T. Vu, and W. C. Stwalley, J. Mol. Spectrosc. 85, 150 (1981).

[45] T. Szidarovszky, A. G. Császár, and G. Czakó, Phys. Chem. Chem. Phys. 12, 8373 (2010).

[46] H.-L. Dai and R. W. Field, Molecular Dynamics and Spectroscopy by Stimulated Emission Pumping (World Scientific, Singapore, 1995).

[47] A. Leclerc, D. Viennot, G. Jolicard, R. Lefebvre, and O. Atabek, Phys. Rev. A 94, 043409 (2016).

[48] C. Fábri, R. Marquardt, A. G. Császár, and M. Quack, J. Chem. Phys. 150, 014102 (2019).

[49] Y. Zhang, E. Lötstedt, and K. Yamanouchi, J. Phys. B 50, 185603 (2017). 